\title{
On the minimum and maximum mass of neutron stars and the delayed collapse
}

\author{
K. Strobel and M. K. Weigel \\ Sektion Physik, Ludwig-Maximilians Universität München, Am Coulombwall 1, 85748 Garching, Germany \\ Received 23 September 2000 / Accepted 28 November 2000

\begin{abstract}
The minimum and maximum mass of protoneutron stars and neutron stars are investigated. The hot dense matter is described by relativistic (including hyperons) and non-relativistic equations of state. We show that the minimum mass $\left(\sim 0.88-1.28 M_{\odot}\right)$ of a neutron star is determined by the earliest stage of its evolution and is nearly unaffected by the presence of hyperons. The maximum mass of a neutron star is limited by the protoneutron star or hot neutron star stage. Further we find that the delayed collapse of a neutron star into a black hole during deleptonization is not only possible for equations of state with softening components, as for instance, hyperons, meson condensates etc., but also for neutron stars with a pure nucleonic-leptonic equation of state.
\end{abstract}

Key words. stars: evolution - stars: neutron - dense matter - equation of state

\section{Introduction}

It is expected that a neutron star (NS) is born as a result of the gravitational collapse of the iron core of a massive $\left(M>8 M_{\odot}\right)$ evolved star (e.g. Bethe 1990). Shortly after core bounce (some $10 \mathrm{~ms}$ ) a hot, lepton rich NS, called protoneutron star (PNS), is formed. This PNS evolves in some minutes into a cold, lepton pure NS due to the loss of neutrinos (e.g. Burrows \& Lattimer 1986; Keil \& Janka 1995; Pons et al. 1999). During these first minutes the PNS can collapse delayed to a black hole (BH) if its initial mass is high enough, either due to the loss of neutrinos or due to post bounce accretion.

The aim of this work is to study the minimum and the maximum mass of NSs and the possibility of $\mathrm{BH}$ formation during the deleptonization period. Recently Gondek et al. (1997, 1998), Goussard et al. (1998) and Strobel et al. (1999a) have calculated limits on the minimum mass of NSs, using equations of state (EOSs) including nucleons $(n, p)$ and leptons. In this paper refinements to these approaches are given by calculating PNSs for a large sample of EOSs using a non-relativistic Thomas-Fermi model (Myers \& Świạtecki 1996; Strobel et al. 1999b,a) and a relativistic Hartree model (Serot \& Walecka 1986; Schäfer 1997) including hyperons. Limits on the maximum mass of NSs were studied by numerous authors (e.g. Takatsuka 1995; Bombaci 1996; Ellis et al. 1996; Prakash et al. 1997; Gondek et al. 1998) using different kinds of EOSs.

Send offprint requests to: K. Strobel, e-mail: Klaus.Strobel@physik.uni-muenchen.de
All these investigations start with models of PNSs about 1-3 s after core bounce (see Sect. 2 for more details). In this paper the earliest stage of a PNS is taken additionally into account, which is reached about $100 \mathrm{~ms}$ after core bounce, to calculate the maximum mass of a NS (see Sect. 2 for more details).

\section{Inside a protoneutron star}

As pointed out in the Introduction a special topic of this investigation is the more detailed incorporation of the earliest stage of the PNS, about $0.1-1 \mathrm{~s}$ after core bounce (Burrows \& Lattimer 1986; Burrows et al. 1995; Keil et al. 1996; Pons et al. 1999). This early type PNS is characterized by a hot shocked envelope with an entropy per baryon of $s \sim 4-6$ (in units of the Boltzmann constant, $k_{\mathrm{B}}$ ) for baryon number densities $n<n_{\text {env }}$, an unshocked core with $s \sim 1-2$ for densities $n>n_{\text {core }}$, and a transition region between these densities (Burrows et al. 1995; Keil et al. 1996). For the description of this early stage of the PNS we use, in accordance with the investigations of Burrows \& Lattimer (1988), Burrows et al. (1995) and Keil et al. (1996), baryon densities in the range of 0.002$0.02 \mathrm{fm}^{-3}$ for $n_{\text {env }}$ and $0.1 \mathrm{fm}^{-3}$ for $n_{\text {core }}$. The entropy per baryon is chosen between $5-6$ in the envelope and between 1-2 in the core, respectively. The investigation is performed for the early type PNS models with trapped neutrinos using a constant lepton number $\left(Y_{1}=0.4\right)$ for densities above $n=610^{-4} \mathrm{fm}^{-3}$.

We assume that post bounce accretion onto the protoneutron star stops within the first $500 \mathrm{~ms}$ after core 
Table 1. Properties of cold symmetric nuclear matter. The entries are: energy per baryon, $u$; saturation density, $n_{0}$; incompressibility, $K_{\infty}$; symmetry energy, $J$; effective nucleon mass, $m^{*} / m$

\begin{tabular}{lccccc}
\hline & $\begin{array}{c}u \\
{[\mathrm{MeV}]}\end{array}$ & $\begin{array}{c}n_{0} \\
{\left[\mathrm{fm}^{-3}\right]}\end{array}$ & $\begin{array}{c}K_{\infty} \\
{[\mathrm{MeV}]}\end{array}$ & $\begin{array}{c}J \\
{[\mathrm{MeV}]}\end{array}$ & $m^{*} / m$ \\
\hline TF & -16.24 & 0.161 & 234 & 32.7 & 0.87 \\
GM1 & -16.30 & 0.153 & 300 & 32.5 & 0.70 \\
GM3 & -16.30 & 0.153 & 240 & 32.5 & 0.78 \\
NL1 & -16.42 & 0.152 & 212 & 43.5 & 0.57 \\
\hline
\end{tabular}

bounce. The amount of accreted matter, and the time at which the accretion through the shock front stops are still open questions. The amount of accreted matter after core bounce ranges from $0.5 M_{\odot}$ during the first $10 \mathrm{~ms}$ or so to 0.001 to $0.15 M_{\odot}$ during the following $100 \mathrm{~ms}$ or so, but in most calculations significant accretion stops about $500 \mathrm{~ms}$ after core bounce (for a further discussion of this topic see e.g. Burrows \& Lattimer 1988; Chevalier 1989; Herant et al. 1992; Burrows et al. 1995; Janka \& Müller 1996; Mezzacappa et al. 1998; Fryer \& Heger 2000).

About 1-3 s after core bounce the PNS has a nearly constant entropy per baryon $(s \sim 2)$ and an approximately constant lepton number $\left(Y_{1} \sim 0.4\right)$ for densities $n>610^{-4} \mathrm{fm}^{-3}$. The next stage in the lifetime of a NS is reached after about $10-30 \mathrm{~s}$, where the hot NS $(s \sim 2)$ is now transparent to neutrinos. During the following minutes this hot NS evolves into a cold NS. For a more detailed description of the evolution of PNSs see, for instance, Prakash et al. (1997) and Strobel et al. (1999a).

The EOSs used in this paper for the description of PNSs are: (i) A non-relativistic Thomas-Fermi model (TF) for finite temperatures developed by Strobel et al. (1999a,b). (ii) A relativistic Hartree model for finite temperatures developed by Schäfer (1997). The Hartree model includes the following hyperons and nucleonic isobars: $\Lambda, \Sigma^{-}, \Sigma^{0}, \Sigma^{+}, \Xi^{-}, \Xi^{0}, \Delta^{-}, \Delta^{0}, \Delta^{+}, \Delta^{++}$(the Delta resonances are not included in the NL1 model).

The main properties of cold symmetric nuclear matter of the different models are listed in Table 1 (see Strobel et al. 1999a; Glendenning \& Moszkowski 1991; Reinhard 1989). The coupling constants for the Hartree models (GM1, GM3, NL1) are listed in Table 2. We take the nucleon-hyperon coupling strength equal to the nucleonnucleon coupling strength for simplicity. This restriction will not change the results for the minimum mass of a NS, as we will show later. The influence on the maximum mass will be strong, but for this point we refer to Huber et al. (1998), where this question is studied for a large spectrum of different coupling constants and different nucleon-hyperon coupling strengths for cold NSs. The EOSs for the different models with nucleonic-leptonic matter (TF, GM1, GM3 and NL1) and nuclear matter including hyperons $\left(\mathrm{GM}_{\mathrm{Hyp}}, \mathrm{GM} 3_{\mathrm{Hyp}}\right.$ and $\left.\mathrm{NL} 1_{\mathrm{Hyp}}\right)$ are shown in Figs. 1 and 2. The EOSs for baryon number densities
Table 2. Coupling constants for the relativistic Hartree models. The coupling constants are given for the following meson masses: $m_{\sigma}=550 \mathrm{MeV}, m_{\omega}=783 \mathrm{MeV}$ and $m_{\rho}=770 \mathrm{MeV}$ (the coupling constants are converted according to these meson masses)

\begin{tabular}{lccccc}
\hline & $g_{\sigma}^{2} / 4 \pi$ & $g_{\omega}^{2} / 4 \pi$ & $g_{\rho}^{2} / 4 \pi$ & $10^{3} \bar{b}$ & $10^{3} \bar{c}$ \\
\hline GM1 & 7.288 & 8.959 & 5.346 & 2.947 & -1.070 \\
GM3 & 6.139 & 6.041 & 5.807 & 8.659 & -2.421 \\
NL1 & 10.2099 & 13.6108 & 8.0260 & 2.4578 & -3.4334 \\
\hline
\end{tabular}

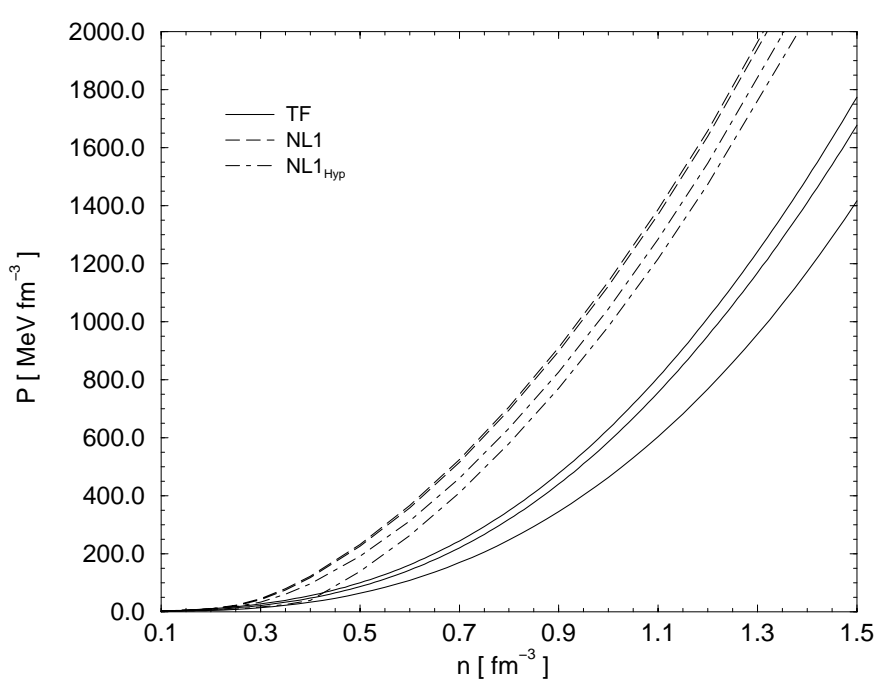

Fig. 1. Pressure versus baryon number density in the high density region for different EOSs. The solid curves correspond (from the bottom to the top) to the cold EOS, the $s=1, Y_{1}=0.4 \mathrm{EOS}$ and the $s=2, Y_{1}=0.4 \mathrm{EOS}$ of the TF model. The long dashed curves correspond to the cold EOS (lower) and the $s=1, Y_{1}=0.4$ EOS (upper) of the NL1 model without hyperons (dot-dashed lines, hyperons included)

below $n=0.1 \mathrm{fm}^{-3}$ are taken from the investigation of Strobel et al. (1999a).

The NL1 coupling constants lead to the stiffest EOSs at high densities, since the high density behaviour is dominated by the strength of the $\omega$-coupling. However the incompressibility is the smallest. In this respect one has to keep in mind that the incompressibility determines the stiffness only near saturation and for higher densities the second and third derivatives of the incompressibility are important for the stiffness (see for instance Ramschütz et al. 1990).

\section{The minimum mass of neutron stars}

The minimum and maximum mass of non-rotating NSs for the different EOSs and different choices of the transition region between the hot shocked envelope and the unshocked core of the PNS are given in Table 3. It turned out that the minimum mass of a NS is determined by the earliest stage of the lifetime of a PNS. The values for the minimum gravitational (baryonic) mass, $M_{\mathrm{G}}\left(M_{\mathrm{B}}\right)$, lie in the range of $0.878-1.284 M_{\odot}\left(0.949-1.338 M_{\odot}\right)$ for 
Table 3. EOSs used in this paper and resulting minimum and maximum masses. The entries are: time after core bounce, $t$; entropy per baryon in the envelope, $s_{\text {env }}$; entropy per baryon in the core, $s_{\text {core }}$; maximum baryon number density of the envelope correlated with the entropy per baryon in the envelope, $n_{\text {env }}\left(s_{\text {env }}\right)$; minimum baryon number density of the core correlated with the entropy per baryon in the core, $n_{\text {core }}\left(s_{\text {core }}\right)$; minimum baryonic mass of the PNS, $M_{\mathrm{B}}^{\mathrm{min}}$; resulting minimum gravitational mass of the cold NS, $M_{\mathrm{G}}^{\min }(T=0)$; maximum baryonic mass of the PNS or NS, $M_{\mathrm{B}}^{\max }$; maximum gravitational mass of the cold NS determined by the PNS or hot NS, $M_{\mathrm{G}}^{\max }(T=0)$; possibility of the formation of a black hole during deleptonization, BH

\begin{tabular}{|c|c|c|c|c|c|c|c|c|c|c|}
\hline Model & $\begin{array}{c}t \\
{[\mathrm{~s}]}\end{array}$ & $s_{\text {env }}$ & $s_{\text {core }}$ & $\begin{array}{c}n_{\mathrm{env}}\left(s_{\mathrm{env}}\right) \\
{\left[\mathrm{fm}^{-3}\right]}\end{array}$ & $\begin{array}{c}n_{\text {core }}\left(s_{\text {core }}\right) \\
{\left[\mathrm{fm}^{-3}\right]}\end{array}$ & $\begin{array}{l}M_{\mathrm{B}}^{\min } \\
{\left[M_{\odot}\right]}\end{array}$ & $\begin{array}{c}M_{\mathrm{G}}^{\min }(T=0) \\
{\left[M_{\odot}\right]}\end{array}$ & $\begin{array}{l}M_{\mathrm{B}}^{\max } \\
{\left[M_{\odot}\right]}\end{array}$ & $\begin{array}{c}M_{\mathrm{G}}^{\max }(T=0) \\
{\left[M_{\odot}\right]}\end{array}$ & $\mathrm{BH}$ \\
\hline \multirow[t]{8}{*}{$\mathrm{TF}$} & $0.1-1$ & 6.0 & 2.0 & 0.002 & 0.1 & 1.338 & 1.284 & 2.366 & - & yes \\
\hline & $0.1-1$ & 6.0 & 1.0 & 0.002 & 0.1 & 0.949 & 0.878 & 2.371 & - & yes \\
\hline & $0.1-1$ & 6.0 & 1.0 & 0.005 & 0.1 & 1.117 & 1.021 & 2.372 & - & yes \\
\hline & $0.1-1$ & 5.0 & 1.0 & 0.01 & 0.1 & 1.020 & 0.940 & 2.372 & - & yes \\
\hline & $0.1-1$ & 5.0 & 1.0 & 0.02 & 0.1 & 1.229 & 1.114 & 2.374 & - & yes \\
\hline & $1-3$ & 2.0 & 2.0 & - & - & - & - & 2.364 & 1.969 & - \\
\hline & $10-30$ & 2.0 & 2.0 & - & - & - & - & 2.449 & - & - \\
\hline & $\infty$ & 0.0 & 0.0 & - & - & - & - & 2.417 & - & - \\
\hline \multirow[t]{6}{*}{ GM1 } & $0.1-1$ & 6.0 & 1.0 & 0.005 & 0.1 & 1.255 & 1.206 & 2.629 & - & yes \\
\hline & $0.1-1$ & 5.0 & 1.0 & 0.005 & 0.1 & 1.019 & 0.988 & 2.628 & - & yes \\
\hline & $0.1-1$ & 5.0 & 1.0 & 0.01 & 0.1 & 1.140 & 1.100 & 2.629 & - & yes \\
\hline & $1-3$ & 2.0 & 2.0 & - & - & - & - & 2.613 & 2.251 & - \\
\hline & $10-30$ & 2.0 & 2.0 & - & - & - & - & 2.670 & - & - \\
\hline & $\infty$ & 0.0 & 0.0 & - & - & - & - & 2.706 & - & - \\
\hline \multirow[t]{6}{*}{$\mathrm{GM}_{\mathrm{Hyp}}$} & $0.1-1$ & 6.0 & 1.0 & 0.005 & 0.1 & 1.255 & 1.206 & 2.462 & - & yes \\
\hline & $0.1-1$ & 5.0 & 1.0 & 0.005 & 0.1 & 1.019 & 0.987 & 2.461 & - & yes \\
\hline & $0.1-1$ & 5.0 & 1.0 & 0.01 & 0.1 & 1.140 & 1.100 & 2.462 & - & yes \\
\hline & $1-3$ & 2.0 & 2.0 & - & - & - & - & 2.401 & - & yes \\
\hline & $10-30$ & 2.0 & 2.0 & - & - & - & - & 2.304 & 2.015 & - \\
\hline & $\infty$ & 0.0 & 0.0 & - & - & - & - & 2.353 & - & - \\
\hline \multirow[t]{6}{*}{ GM3 } & $0.1-1$ & 6.0 & 1.0 & 0.005 & 0.1 & 1.255 & 1.205 & 2.204 & 1.939 & no \\
\hline & $0.1-1$ & 5.0 & 1.0 & 0.005 & 0.1 & 1.039 & 1.005 & 2.203 & 1.938 & no \\
\hline & $0.1-1$ & 5.0 & 1.0 & 0.01 & 0.1 & 1.145 & 1.104 & 2.204 & 1.939 & no \\
\hline & $1-3$ & 2.0 & 2.0 & - & - & - & - & 2.212 & - & - \\
\hline & $10-30$ & 2.0 & 2.0 & - & - & - & - & 2.249 & - & - \\
\hline & $\infty$ & 0.0 & 0.0 & - & - & - & - & 2.247 & - & - \\
\hline \multirow[t]{6}{*}{ GM3 $3_{\text {Hyp }}$} & $0.1-1$ & 6.0 & 1.0 & 0.005 & 0.1 & 1.255 & 1.205 & 2.047 & - & yes \\
\hline & $0.1-1$ & 5.0 & 1.0 & 0.005 & 0.1 & 1.039 & 1.005 & 2.046 & - & yes \\
\hline & $0.1-1$ & 5.0 & 1.0 & 0.01 & 0.1 & 1.145 & 1.104 & 2.047 & - & yes \\
\hline & $1-3$ & 2.0 & 2.0 & - & - & - & - & 2.015 & - & yes \\
\hline & $10-30$ & 2.0 & 2.0 & - & - & - & - & 1.898 & 1.699 & - \\
\hline & $\infty$ & 0.0 & 0.0 & - & - & - & - & 1.911 & - & - \\
\hline \multirow[t]{4}{*}{ NL1 } & $0.1-1$ & 5.0 & 1.0 & 0.005 & 0.1 & 1.175 & 1.111 & 3.218 & - & yes \\
\hline & $1-3$ & 2.0 & 2.0 & - & - & - & - & 3.162 & 2.663 & (yes) \\
\hline & $10-30$ & 2.0 & 2.0 & - & - & - & - & 3.202 & $(2.689)$ & - \\
\hline & $\infty$ & 0.0 & 0.0 & - & - & - & - & 3.297 & - & - \\
\hline \multirow[t]{4}{*}{$\mathrm{NL1}_{\mathrm{Hyp}}$} & $0.1-1$ & 5.0 & 1.0 & 0.005 & 0.1 & 1.169 & 1.104 & 3.017 & - & yes \\
\hline & $1-3$ & 2.0 & 2.0 & - & - & - & - & 2.942 & - & yes \\
\hline & $10-30$ & 2.0 & 2.0 & - & - & - & - & 2.871 & 2.399 & - \\
\hline & $\infty$ & 0.0 & 0.0 & - & - & - & - & 2.962 & - & - \\
\hline
\end{tabular}

the different models (see Table 3). They are larger by a factor of ten than the minimum mass of a cold NS (e.g. Strobel et al. 1997) emerging from a calculation for the minimum mass of the cold NS based only on the solution of the Tolman-Oppenheimer-Volkoff equation with the corresponding EOS for cold NS matter. This shift due to the properties of early type PNSs was also recently found by Goussard et al. (1998) and Strobel et al. (1999a). Gondek et al. $(1997,1998)$ found an even smaller value for the minimum mass $\left(M_{\mathrm{G}} \sim 0.6 M_{\odot}\right)$. The reason for this is that they use models for PNSs about $1-3 \mathrm{~s}$ after core bounce, where the minimum mass is lower, see 


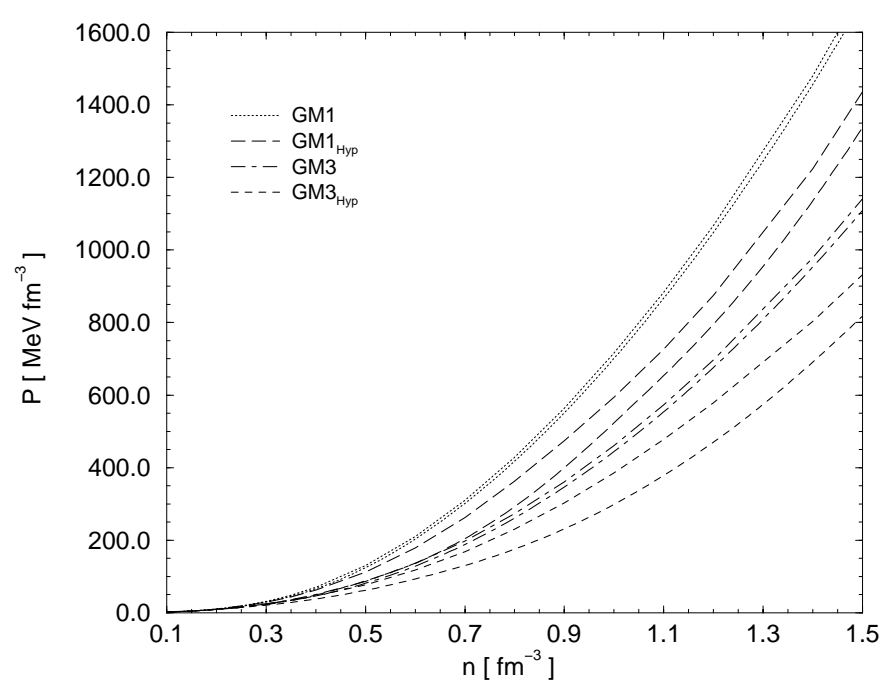

Fig. 2. Pressure versus baryon number density in the high density region for different EOSs. The dotted curves correspond to the cold EOS (lower) and the $s=1, Y_{1}=0.4$ EOS (upper) of the GM1 model without hyperons (long dashed lines, hyperons included). The dot-dashed curves correspond to the cold EOS (lower) and the $s=1, Y_{1}=0.4$ EOS (upper) of the GM3 model without hyperons (short dashed lines, hyperons included)

also Strobel et al. (1999a). Our models with hyperons included do not change the values for the minimum mass significantly $\left(\Delta M_{\mathrm{G}} \leq 0.007 M_{\odot}\right)$. This result could be expected since the maximum baryon number density inside the core of such a NS is smaller than two times the nuclear matter density and hence the hyperon fraction is rather small.

Let us now turn to the question, why there is a minimum mass in this early stage of the evolution. A star becomes dynamically unstable if its mean adiabatic index, $\bar{\Gamma}$, lies below $4 / 3$. In the case of a cold NS this is caused by the neutron drip at a density of $n \sim 2.610^{-4} \mathrm{fm}^{-3}$. For the earliest stage of the PNS the drop below $\bar{\Gamma}=4 / 3$ is caused by the entropy drop between the hot shocked envelope and the unshocked core of the PNS. For a complete description of the stability criteria of neutron stars see Shapiro \& Teukolsky (1983).

\section{The maximum mass of neutron stars and the possibility of black hole formation during deleptonization}

Quite interesting is the result for the maximum gravitational mass for NSs, for which we obtain values in the range between $1.699-2.663 M_{\odot}$ (see Table 3 ) for the different EOSs used in this paper, with the possibility of the formation of a $\mathrm{BH}$ during deleptonization for EOSs with a pure nucleonic-leptonic composition and EOSs which include hyperons. For instance, for the NL1 EOS, the maximum baryonic mass for the first milliseconds, $M_{\mathrm{B}}^{\max }=3.218 M_{\odot}$, is larger than the allowed maximum baryonic mass of $3.162 M_{\odot}$ after $1-3 \mathrm{~s}$, which implies

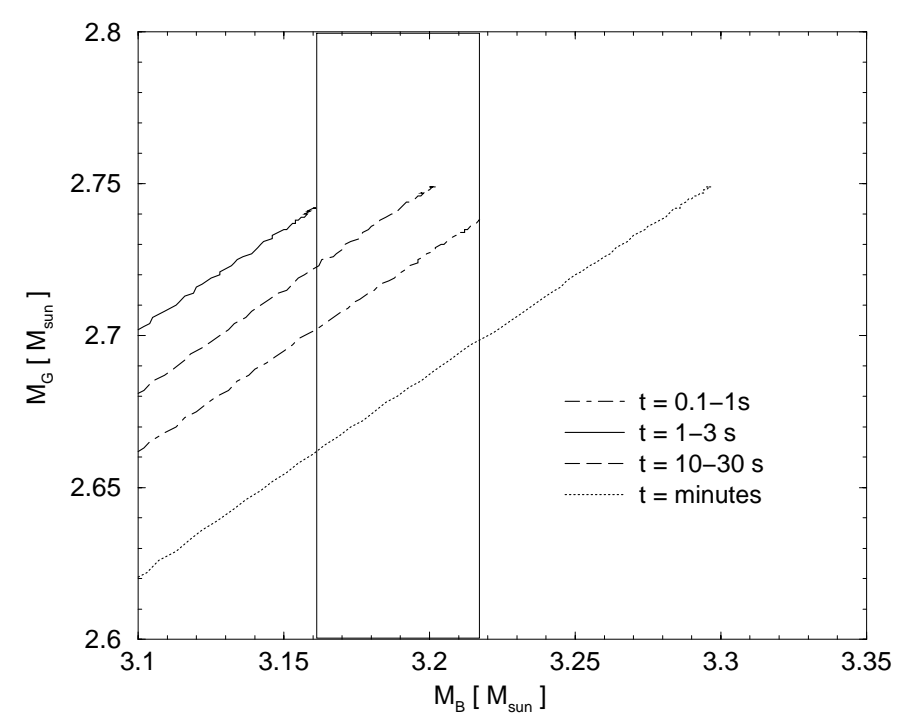

Fig. 3. Maximum gravitatinal versus maximum baryonic mass for different evolutionary stages of the NL1 EOSs. The two lines denote the mass window for the delayed collapse

the possibility of a BH formation (see Table 3 and Fig. 3). This value also sets the boundary of $2.663 M_{\odot}$ for the maximum gravitational mass for the cold NS. The maximum baryonic mass of the first stage is even larger than maximum value 10-30 s after core bounce fore the NL1 EOS. This means that the delayed collapse is also possible for the totally deleptonized hot NS of the NL1 EOS. It should be mentioned in this context that the exact entropy profile of the hot shocked envelope of an early type PNS plays a minor role in determining the maximum baryonic mass, since a PNS with a constant entropy per baryon of $s=1$ throughout the whole PNS and a lepton number of $Y_{1}=0.4$ leads to nearly the same maximum baryonic mass (e.g. for the NL1 model: $M_{\mathrm{B}}^{\max }=3.218 M_{\odot}$, $0.1-1.0 \mathrm{~s}$ after core bounce; $M_{\mathrm{B}}^{\max }=3.219 M_{\odot}$, for an entropy per baryon of $s=1$ throughout the whole PNS and $\left.Y_{1}=0.4\right)$. This means that the maximum mass depents nearly complete on the properties of the core of the $\mathrm{PNS}^{1}$.

Similar behaviour is found for the other EOSs with exception of the nucleonic-leptonic EOS GM3, where the allowed maximum mass after $1-3$ s exeeds the maximum masses of the first period. The reason why this possibility of a $\mathrm{BH}$ formation was dismissed so far for a pure nucleonic-leptonic composition (e.g. Takatsuka 1995; Bombaci 1996; Ellis et al. 1996; Prakash et al. 1997; Gondek et al. 1998) was that these authors start their calculations about 1-3 s after core bounce, since the following stages allow only larger baryonic masses. One should mention in this context that for the nucleonic-leptonic EOSs the differences in the maximum masses in the first seconds are more than three times smaller ${ }^{2}\left(<0.056 M_{\odot}\right)$ than for

1 Note: Other properties of an early type PNS (e.g. the radius) are strongly affected by the entropy profile.

${ }^{2}$ For that reason the effect was overlooked in Strobel et al. (1999a). 


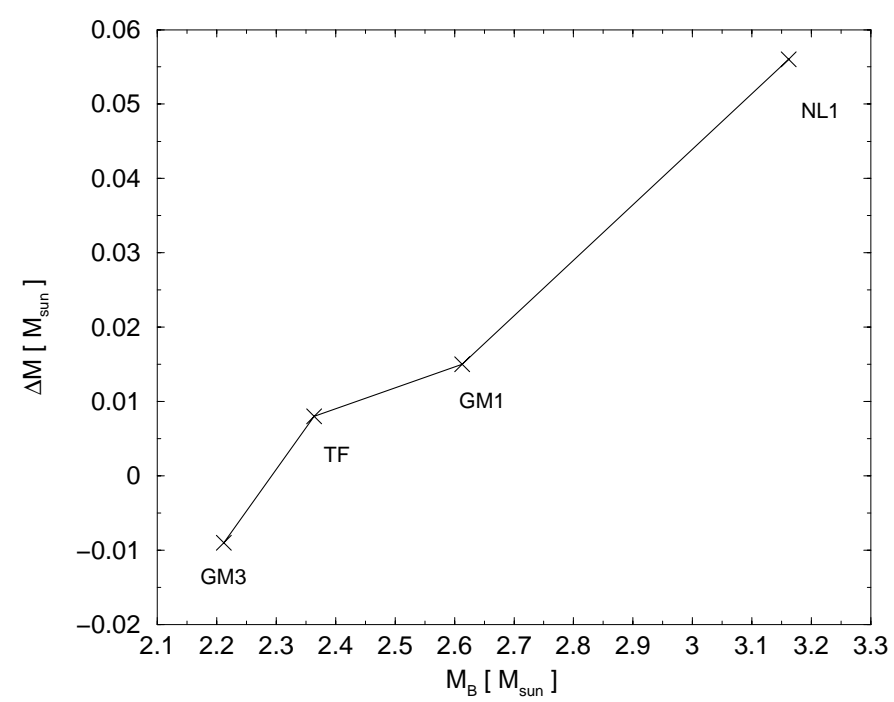

Fig. 4. Baryonic mass difference between the early type PNS stage (0.1-1 s after core bounce) and the late type PNS stage ( $1-3$ s after core bounce) versus resulting maximum baryonic mass for the different pure nucleonic-leptonic EOSs

the softer EOSs with hyperons $\left(0.146-0.158 M_{\odot}\right)$. Due to this, for the EOSs including hyperons the possibility of a $\mathrm{BH}$ formation shows up even by starting the calculation 1-3 s after core bounce (see Table 3). It turned out, that the mass window for the delayed collapse of the pure nucleonic-leptonic EOSs is an increasing function of the maximum possible mass of the different models (see Fig. 4). The EOSs including hyperons did not show a similar behaviour, the mass window is nearly constant for all used EOSs. The minimum value of the maximum baryonic mass for the models including hyperons is reached by the hot NS without trapped neutrinos (10-30 s after core bounce). This, as already mentioned, can lead to a delayed collapse of the NS during deleptonization if the initial mass of the PNS is high enough (e.g. Baumgarte et al. 1996; Pons et al. 1999).

It is an open question how large the maximum mass of a NS really is. It is clear that an EOS should allow NS masses larger than $1.4 M_{\odot}$ since measurements of pulsars in binary systems show up values around this mass (e.g. Thorsett \& Chakrabarty 1999). If quasi periodic oscillations (e.g. van der Klis 2000) are taken into account, the possible maximum mass of an EOS has to be larger than 1.8-2.0 $M_{\odot}$ (e.g. Schaab \& Weigel 1999). This whould lead to the conclusion that only the GM1 and the NL1 EOSs (with and without hyperons) are stiff enough to allow NSs with a larger mass.

\section{Discussion and conclusion}

In this paper we presented a calculation of the minimum and maximum mass of a NS taking the early evolution, shortly after core bounce, into account. We found that the minimum mass of a NS is limited by the earliest stage of its evolution ( $\sim 0.1-1 \mathrm{~s}$ after core bounce). Therefore the minimum gravitational mass has values between 0.878 and $1.284 M_{\odot}$. These values lie in the same range as those found in recent works by Goussard et al. (1998) and Strobel et al. (1999a). Additionally we have examined the influence of hyperons on the minimum mass. As expected it turned out that the minimum mass is nearly unaffected by the inclusion of hyperons. Another possibility of NS production is the accretion induced collapse of a white dwarf (Canal \& Schatzman 1976), but the numerical simulation of Woosley \& Baron (1992) showed that the resulting NS will have a mass of $\sim 1.4 M_{\odot}$ since the white dwarf collapses without significant mass loss after reaching the maximum mass of a white dwarf. From the results reported it is obvious that NSs, which are born in type-II supernovae or due to accretion induced collapse of a white dwarf, have a minimum mass about ten times larger than the possible minimum mass of a cold NS $\left(\sim 0.1 M_{\odot}\right)$.

With respect to the maximum mass of a NS calculated by solving the Tolman-Oppenheimer-Volkoff equation for NS matter the outcome is rather uncertain, since the EOS in the high density region rests strongly on theoretical extrapolations. The underlying model, for instance, different many-body approximations and/or inclusion of more massive baryons, quarks, condensates etc., can render the results for the maximum masses significantly (e.g. Prakash et al. 1997; Huber et al. 1998). However, as a result of our work it turned out, that the maximum mass of a NS is limited by the first $\sim 30 \mathrm{~s}$ of its evolution. The maximum baryonic mass of a NS, constructed with pure nucleonicleptonic EOSs and EOSs including hyperons, is always reached during this early epoch of the evolution.

Finally we found that $\mathrm{BH}$ formation during deleptonization is also possible for NSs with pure nucleonicleptonic EOSs if the initial mass is high enough. This property of NSs was expected till now to be possible only for EOSs including softening ingredients, like hyperons, meson condensates or a quark-hadron phase transition (e.g. Brown \& Bethe 1994; Glendenning 1995). There are numerous papers on this topic (e.g. Takatsuka 1995; Bombaci 1996; Ellis et al. 1996; Prakash et al. 1997; Gondek et al. 1998), but all these investigations started their calculations about $1-3 \mathrm{~s}$ after core bounce. In this paper we included the earliest stage and found that the maximum baryonic mass of the early type PNSs $(\sim 0.1-1$ s after core bounce) could be up to $0.056 M_{\odot}$ larger than that of the late type PNS ( 1-3 s after core bounce). Due to this result it seems possible that a NS with a pure nucleonicleptonic EOS could also undergo delayed collapse into a $\mathrm{BH}$ during the first seconds of its evolution. This question could only be finally decided in time evolution calculations like this including softening ingredients (e.g. Baumgarte et al. 1996), since we have used approximate values for the entropy per baryon and the lepton number at fixed times. Post bounce accretion (e.g. Chevalier 1989; Herant et al. 1992) could lead also to a delayed collapse, but it is uncertain till now how much matter falls through the shock front within the first second after core bounce, so that if a cut off of a neutrino signal after a few seconds 
is detected in the future, the two ways of $\mathrm{BH}$ formation could possibly be indistinguishable.

Acknowledgements. We want to thank A. Schäfer for providing us with his program. We want to thank H.-Th. Janka and Ch. Schaab for many helpful discussions. One of us, K. S., gratefully acknowledges the Bavarian State for financial support.

\section{References}

Baumgarte, T. W., Janka, H. T., Keil, W., Shapiro, S. L., \& Teukolsky, S. A. 1996, ApJ, 468, 823

Bethe, H. A. 1990, Rev. Mod. Phys., 62, 801

Bombaci, I. 1996, ApJ, 305, 871

Brown, G. E., \& Bethe, H. A. 1994, ApJ, 423, 659

Burrows, A., \& Lattimer, J. M. 1986, ApJ, 307, 178

Burrows, A., \& Lattimer, J. M. 1988, Phys. Rep., 163, 51

Burrows, A., Hayes, J., \& Fryxell, B. A. 1995, ApJ, 450, 830

Canal, R., \& Schatzman, E. 1976, A\&A, 46, 229

Chevalier, R. A. 1989, ApJ, 346, 847

Ellis, P. J., Lattimer, J. M., \& Prakash, M. 1996, Comments Nucl. Part. Phys., 22, 63

Fryer, C. L., \& Heger, A. 2000, ApJ, 541, 1033

Glendenning, N. K. 1995, ApJ, 448, 797

Glendenning, N. K., \& Moszkowski, S. A. 1991, Phys. Rev. Lett., 67, 2414

Gondek, D., Haensel, P., \& Zdunik, J. L. 1997, A\&A, 325, 217

Gondek, D., Haensel, P., \& Zdunik, J. L. 1998, in ASP Conf. Ser. 138, 1997, Pacific Rim Conference on Stellar Astrophysics, ed. K. L Chan, K. S. Cheng, \& H. P. Singh, 131

Goussard, J. O., Haensel, P., \& Zdunik, J. L. 1998, A\&A, 330, 1005
Herant, M., Benz, W., \& Colgate, S. 1992, ApJ, 395, 642

Huber, H., Weber, F., Weigel, M. K., \& Schaab, C. 1998, Int. J. Mod. Phys. E, 7, 301

Janka, H. T., \& Müller, E. 1996, A\&A, 306, 167

Keil, W., \& Janka, H. T. 1995, A\&A, 296, 145

Keil, W., Janka, H. T., \& Müller, E. 1996, ApJ, 473, L111

Mezzacappa, A., Calder, A. C., Bruenn, S. W., et al. 1998, ApJ, 495, 911

Myers, W. D., \& Świątecki, W. J. 1996, Nucl. Phys. A, 601, 141

Pons, J. A., Reddy, S., Prakash, M., Lattimer, J. M., \& Miralles, J. A. 1999, ApJ, 513, 780

Prakash, M., Bombaci, I., Prakash, M., et al. 1997, Phys. Rep., 280,1

Ramschütz, J., Weber, F., \& Weigel, M. K. 1990, J. Phys. G, 16, 987

Reinhard, P. G. 1989, Rep. Prog. Phys., 52, 439

Schaab, C., \& Weigel, M. K. 1999, MNRAS, 308, 718

Schäfer, A. 1997, Eigenschaften der Materie heißer Protoneutronensterne, Master's thesis, Universität München, unpublished

Serot, B. D., \& Walecka, J. D. 1986, Adv. Nucl. Phys., 16, 1

Shapiro, S. L., \& Teukolsky, S. A. 1983, Black Holes, White Dwarfs and Neutron Stars (John Wiley \& Sons, New York) Strobel, K., Weber, F., Schaab, C., \& Weigel, M. K. 1997, Int. J. Mod. Phys. E, 6, 669

Strobel, K., Schaab, C., \& Weigel, M. K. 1999a, A\&A, 350, 497

Strobel, K., Weber, F., \& Weigel, M. K. 1999b, Z. Naturforsch., $54 a, 83$

Takatsuka, T. 1995, Nucl. Phys. A, 588, 365

Thorsett, S. E., \& Chakrabarty, D. 1999, ApJ, 512, 288

van der Klis, M. 2000, ARA\&A, 38, 717

Woosley, S. E., \& Baron, E. 1992, ApJ, 391, 228 